\title{
Rapid Adsorption Removal of Triclosan and p- chloro-m-xylenol In Water By Nitrogen Doped Magnetic Porous Carbon
}

\section{Qiuxing Li}

Chengdu University of Technology

\section{Li Huang}

Chengdu University of Technology

\section{Paijin Zhu}

Chengdu University of Technology

\section{Min Zhong}

Chengdu University of Technology

Shuxia Xu ( $\nabla$ xushux2013@163.com )

Chengdu University of Technology https://orcid.org/0000-0002-4235-7847

\section{Research Article}

Keywords: Magnetic porous carbon, Nitrogen doping, Phenolic fungicides, Adsorption, Kinetics, Thermodynamics

Posted Date: December 6th, 2021

DOI: https://doi.org/10.21203/rs.3.rs-1037896/v1

License: (c) (i) This work is licensed under a Creative Commons Attribution 4.0 International License. Read Full License 


\section{Abstract}

Contamination of water resources with organic substances like phenolic fungicides is undesirable due to the improvement of living standards, huge production and consumption of daily chemicals, and an increase in the population. In this study, ZIF-67(Co) was synthesized using the "one pot method", and the Co-magnetic porous carbon (Co-NPC) was prepared by ZIF-67 (Co) carbonization in an atmosphere of $\mathrm{N}_{2}$. The materials were tested using a X-ray diffractometer (XRD), scanning electron microscope (SEM), infrared spectroscopy (IR), Raman spectroscopy, X-ray photoelectron spectroscopy (XPS), $\mathrm{N}_{2}$ adsorptiondesorption and magnetization analysis. These methods indicated that the Co-NPC was successfully prepared. With the original morphology of ZIF-67 (Co) crystal, the Co-NPC also has good porosity, magnetic properties and a large specific surface area. In water, Co-NPC-800 has a good adsorption capacity for triclosan (TCS) and p-chloro-m-xylenol (PCMX), which are kinds of aromatic fungicides. The adsorption of Co-NPC-800 on both reached equilibrium within $3 \mathrm{~min}$, which is in accordance with the quasi-second-order kinetic model. At $298 \mathrm{~K}$, the maximum adsorption capacity of Co-NPC-800 for TCS and PCMX was 163 and $39 \mathrm{mg} \cdot \mathrm{g}^{-1}$, respectively. The adsorption of TCS and PCMX by Co-NPC-800 is a spontaneous endothermic process with reduced entropy. The combination of Co-NPC-800 and phenols come from multiple action of electrostatic, $\pi-\pi$ and hydrogen bond effects. Moreover, Co-NPC-800 can be regenerated through simple washing and can be reused several times. Therefore, Co-NPC-800 has great potential to be applied across sewage treatments and other environmental fields.

\section{Introduction}

In recent years, the environment has faced a massive threat due to the continuous growth of the world population, high energy consumption and toxic waste emissions (Bao \&Fang 2012). Various contaminants have been detected in aquatic environments, including pesticide residue, heavy metals, phenolic fungicides amongst others. Phenolic fungicides, widely used in daily chemicals, are usually aromatic phenols (Yoo et al. 2018). Triclosan (TCS) and p-chloro-m-xylenol (PCMX) are two common phenolic fungicides containing chlorine, which is believed to cause continuous pollution to the environment because they contain chlorine atoms ( $\mathrm{Ng}$ et al. 2019). Furthermore, chlorine compounds are harmful to the human body (Tan et al. 2021). As such, the removal of phenolic fungicides from the environment has become a hot research topic (Yu et al. 2020).

In previous studies, there many methods have been used to remove phenolic fungicides in the liquid phase, including biological oxidation (Martín et al. 2008), membrane separation (Diaconu et al. 2010), solid-phase extraction (Mahugo Santana et al. 2009), chemical treatment (Rahmanian et al. 2014), incineration (Oturan et al. 2009), coagulation (Wada et al. 1995) and adsorption (Ahmaruzzaman 2008). In particular, adsorption has been commonly used due to the fact it is simple to operate, requires low energy and is highly effective (Zhang et al. 2015). In the field of environmental science, carbon-based materials including activated carbon, carbon nanotubes, grapheme and biomass-derived carbon have been actively developed for highly efficient absorbency (Apul et al. 2013, Meng et al. 2021). Due to the excellent properties of porous carbon materials, nanoporous carbon materials (NPC) in particular have 
attracted the attention of many researchers (Lee et al. 2004, Yao et al. 2017). NPC have a high specific surface area, which is beneficial for the loading of nanoparticles and the modification of functional groups (Vakili et al. 2021). This leads to improvements in the catalytic performance and adsorption performance of the carbon material(Liu et al. 2015). In order to achieve high adsorption performance, the researchers used all kinds of precursors to prepare the carbon materials including biomass, agricultural residue, industrial waste and metal-organic frameworks (MOFs)(Sule \&Mishra 2020, Teimouri et al. 2019). MOFs are the most widely studied advanced porous materials due to their high specific surface area and porosity. After high-temperature carbonization of MOFs, porous carbon materials can be derived (Chaikittisilp et al. 2013). The porous carbon material prepared with MOFs as template retained the porosity and large specific surface area of MOFs, and was a good adsorbent and catalyst carrier (Wang et al. 2020).

Although MOFs-derived porous carbons are promising materials, their performance can be further improved by introducing dopants such as nitrogen. The most commonly used method to obtain N-doped carbons is to carbonize MOFs loaded with nitrogen-containing compounds (Chen et al. 2018). Bio-MOF-1derived carbon, based on $\mathrm{Zn}_{8}$ (adenine) ${ }_{4}$ (biphenyldicarboxylate) ${ }_{6} \mathrm{O}$, has shown the highest adsorption capability of bisphenol-A (BPA) in water (Bhadra et al. 2018). ZIF-mediated N-doped hollow porous carbon, hardly affected by humic acid, has been used as a high performance adsorbent to remove tetracycline from water (Liang et al. 2020). However, adsorption technology has several downfalls such as having an insufficient capacity development, a high cost and being relatively difficult to recycle (Crini et al. 2019).

In order make carbon materials more easily recyclable, researchers have developed a variety of methods including incremental weight (Kasmi et al. 2017), use of core-shells (Gupta et al. 2014, Lv et al. 2019) and adding magnetism (Oladipo et al. 2018). In N-MCFs (magnetic particle-decorated N-doped carbon foams), it was found that the distorted framework caused by $\mathrm{N}$-doping led to the distortion of graphite lattice and the change in the local density of the carbon atomic state. This significantly contributed to the magnetic moment (Lou et al. 2021). In this work, ZIF-67 (Co), a MOF material with a zeolite structure was prepared. Co-NPC, a porous carbon material mixed with nitrogen elements, was prepared using ZIF-67 (Co) carbonization at a high temperature within a $\mathrm{N}_{2}$ atmosphere. By controlling the carbonization temperature, the cobalt element in the ZIF-67 (Co) framework remained in the porous carbon material obtained, in the form of cobalt oxide and this created the material's magnetism. The resultant magnetic porous carbon material was used for the adsorption and removal of TCS and PCMX in the water. Notably, after nitrogen doping, the material had more adsorption sites and excellent adsorption performance. The magnetic properties of the material facilitated its recycling, which has the potential for broad application within the environmental field.

\section{Experiment}

\subsection{Synthesis of ZIF-67(Co)}


A total of $5.5 \mathrm{~g}$ of 2-methylimidazole and $20 \mathrm{~mL}$ of ultrapure water were added to a $50 \mathrm{~mL}$ beaker, stirred until dissolved and recorded it as solution I. A total of $0.45 \mathrm{~g}$ of $\mathrm{Co}\left(\mathrm{NO}_{3}\right)_{2} \ddot{\mathrm{Y}} 6 \mathrm{H}_{2} \mathrm{O}$ and $3 \mathrm{~mL}$ of ultrapure water were added into a $10 \mathrm{~mL}$ centrifuge tube, dissolved and recorded it as solution II. Solution II was slowly added to solution I and stirred with high speed for $6 \mathrm{~h}$ at room temperature. Subsequently, the centrifuge tube was centrifuged at $8000 \mathrm{rpm}$ for $5 \mathrm{~min}$. Then, the product was taken out and washed alternately with ultrapure water and ethanol. After that, the product was soaked in an ethanol bath at $60^{\circ} \mathrm{C}$ for $6 \mathrm{~h}$, washed with ethanol for 3 times, dried and activated under vacuum at $100^{\circ} \mathrm{C}$ for $24 \mathrm{~h}$.

\subsection{Synthesis of Co-NPCs}

$0.5 \mathrm{~g}$ of the obtained ZIF-67 (Co) sample was placed in a $15 \mathrm{ml}$ porcelain crucible, and then the crucible was calcined in a quartz tube furnace in $\mathrm{N}_{2}$ or $\mathrm{Ar}$ atmosphere. The sample was heated up to $600^{\circ} \mathrm{C}$, $700^{\circ} \mathrm{C}, 800^{\circ} \mathrm{C}$ with $5^{\circ} \mathrm{C} \cdot \mathrm{min}^{-1}$ heating rate and calcined at the target temperature for 3 hours. After it was cooled down, the obtained porous carbon materials (Co-NPCs) were named as Co-NPC-600, Co-NPC-700, Co-NPC-800, Co-NPC-800 (Ar) according to the atmosphere and temperature.

\subsection{Adsorption performance and reusability of Co-NPCs}

$400 \mu \mathrm{L}$ of different materials (Co-NPC-600, Co-NPC-700, Co-NPC-800, Co-NPC-800 (Ar)) suspension, $1 \mathrm{~mL}$ of phosphate buffered saline solution (PBS, $\mathrm{pH}=7.0$ ), $200 \mu \mathrm{L}$ of TCS stock solution and $400 \mu \mathrm{L}$ ultrapure water (or $100 \mu \mathrm{L}$ of PCMX stock solution and $500 \mu \mathrm{L}$ ultrapure water) were added in a $2 \mathrm{~mL}$ centrifugal tube. The adsorption was carried out for $20 \mathrm{~min}$ in a $298 \mathrm{~K}$ water bath. Then, the supernatant was separated with a magnet. The concentrations of TCS/PCMX in the supernatant were analyzed by an UVvisible spectrophotometer at $280 \mathrm{~nm}$. The amount of TCS/PCMX adsorbed on different Co-NPCs were calculated from the expression:

$$
q_{e}=\frac{\left(C_{0}-C_{e}\right) V}{\mathrm{~m}}
$$

Where $q_{e}$ was the amount adsorbed at equilibrium $\left(\mathrm{mg} \cdot \mathrm{g}^{-1}\right), C_{0}$ and $C_{e}$ were the initial and equilibrium concentrations of pollutant in solution $\left(\mu \mathrm{g} \cdot \mathrm{mL}^{-1}\right)$, respectively, $\mathrm{m}$ was the dosage of sorbent $(\mathrm{mg})$, and $\mathrm{V}$ was the volume of the solution $(\mathrm{mL})$.

Washed the separated Co-NPC-800 with ultrapure water and ethanol alternately until TCS or PCMX was no longer detected in the washing solution. Dried the recovered Co-NPC-800 under vacuum for $12 \mathrm{~h}$ at $100^{\circ} \mathrm{C}$. Afterward, repeated the adsorption procedures for 4 times, and calculated the adsorption capacity in different cycle.

\subsection{Adsorption kinetics}

Two conventional kinetic models (quasi-first-order and quasi-second-order) were applied to analyze the experimental data. The quasi-first-order model can be expressed as: 


$$
\ln \left(q_{e}-q_{t}\right)=\ln q_{e}-k_{1} t
$$

Where $q_{e}$ and $q_{t}$ were the adsorbed pollutant amounts on Co-NPC at equilibrium and at various time $t$ $\left(\mathrm{mg} \cdot \mathrm{g}^{-1}\right)$, respectively; $t$ was the adsorption time $(\mathrm{min})$, and $k_{1}$ was the rate constant of the quasi-firstorder model of adsorption $\left(\mathrm{min}^{-1}\right)$. The values of $q_{e}$ and $k_{1}$ could be determined from the intercept and slope of the linear plot of $\ln \left(q_{e}-q_{t}\right)$ versus $t$.

The quasi-second-order model includes all the steps of adsorption including external film diffusion, adsorption, and internal particle diffusion, which is described as:

$$
\frac{\mathrm{t}}{\mathrm{q}_{\mathrm{t}}}=\frac{1}{\mathrm{k}_{2} \mathrm{q}_{\mathrm{e}}^{2}}+\frac{1}{\mathrm{q}_{\mathrm{e}}} \mathrm{t}
$$

Where $q_{e}$ and $q_{t}$ were the adsorption capacity $\left(\mathrm{mg} \cdot \mathrm{g}^{-1}\right)$ at equilibrium and at any time $t(\mathrm{~min})$, respectively; $k_{2}$ was the rate constant of the quasi-second-order model of adsorption $\left(\mathrm{g} \cdot \mathrm{mg}^{-1} \cdot \mathrm{min}^{-1}\right)$. The values of $q_{e}$ and $k_{2}$ were determined from the slope and intercept of the plots of $t / q_{t}$ against $t$.

\subsection{Adsorption thermodynamics}

The thermodynamic parameters such as standard free energy change $\left(\Delta G^{9}\right)$, enthalpy change $\left(\Delta H^{9}\right)$ and entropy change $\left(\Delta S^{9}\right)$ were determined based on the following equations:

$\mathrm{K}=\frac{\mathrm{C}_{\mathrm{s}}}{\mathrm{C}_{\mathrm{e}}}$

$\Delta \mathrm{G}^{0}=-\mathrm{RT} \ln \mathrm{K}$

$$
\begin{aligned}
\ln K_{3}-\ln K_{1} & =\frac{-\Delta \mathrm{H}^{0}}{R}\left(\frac{1}{T_{3}}-\frac{1}{T_{1}}\right) \\
\Delta S^{0} & =\frac{\Delta \mathrm{G}^{0}-\Delta \mathrm{H}^{0}}{\mathrm{~T}}
\end{aligned}
$$

Where $K$ was the adsorption equilibrium constant, $C_{s}$ was the amount of pollutant adsorbed by per mass of Co-NPC-800 ( $\left.\mathrm{mg} \cdot \mathrm{g}^{-1}\right), C_{e}$ was the equilibrium concentration of the target pollutant $\left(\mathrm{mg} \cdot \mathrm{L}^{-1}\right), \mathrm{R}$ was the universal gas constant $\left(8.314 \mathrm{~J} \cdot \mathrm{mol}^{-1} \cdot \mathrm{K}^{-1}\right)$, and T was the absolute temperature $(\mathrm{K})$.

\subsection{Adsorption isotherms}

To further investigate the mechanism of equilibrium adsorption, the results were subjected to Langmuir and Freundlich isothermal models for simulation. The Langmuir model assumes that adsorption occurs on a homogeneous surface by monolayer coverage and no subsequent interaction between adsorbed species. The equation was as follows: 


$$
\frac{C_{e}}{q_{e}}=\frac{C_{e}}{q_{\max }}+\frac{1}{K_{L} q_{\max }}
$$

Where $q_{e}$ was the adsorbed pollutant amount on Co-NPC at equilibrium $\left(\mathrm{mg} \cdot \mathrm{g}^{-1}\right), C_{e}$ were the initial and equilibrium concentrations of pollutant in solution $\left(\mathrm{mg}^{\mathrm{L}} \mathrm{L}^{-1}\right) ; K_{\mathrm{L}}$ was the Langmuir constant $\left(\mathrm{L} \cdot \mathrm{mg}^{-1}\right)$, which was related to the affinity of the binding sites; and $q_{\max }$ represents the maximum adsorption capacity of the adsorbent $\left(\mathrm{mg} \cdot \mathrm{g}^{-1}\right)$. The values of $q_{\max }$ and $K_{L}$ were calculated from the slope and intercept of the linear plot of $C_{e} / q_{e}$ against $C_{e}$.

The Freundlich model was an empirical model based on multilayer adsorption on heterogeneous surfaces. The equation was commonly described as:

$$
\operatorname{lnq}_{\mathrm{e}}=\ln \mathrm{K}_{\mathrm{F}}+\frac{1}{\mathrm{n}} \ln \mathrm{C}_{\mathrm{e}}
$$

Where $q_{e}$ and $C_{e}$ were defined as in the Langmuir isotherm; $K_{F}$ and $n$ were the Freundlich constants that represent the adsorption capacity and adsorption strength, respectively. $K_{F}$ and $n$ could be obtained from the intercept and slope of the linear plot of $\ln q_{e}$ versus $\ln C_{e}$. Moreover, the magnitude of $1 / \mathrm{n}$ quantifies the favorability of adsorption and the degree of heterogeneity of the surface. If $n>1$, suggested favorable adsorption.

\section{Results And Discussion}

\subsection{Characterization of ZIF-67 (Co)}

The lattice data of synthesized ZIF-67 (Co) was analyzed using XRD. As shown in Figure S2, the experimental results conformed to the theory within the literature(Banerjee et al. 2008). The crystal plane diffractions of ZIF-67 (Co) were obtained including 011 (corresponding to $7.31^{\circ}$ ), 002 (corresponding to $10.36^{\circ}$ ), 112 (corresponding to $12.72^{\circ}$ ), 022 (corresponding to $14.40^{\circ}$ ), 013 (corresponding to $16.45^{\circ}$ ), 222 (corresponding to $18.04^{\circ}$ ), 114 (corresponding to $22.15^{\circ}$ ), 233 (corresponding to $24.53^{\circ}$ ), 134 (corresponding to $26.70^{\circ}$ ), 044 (corresponding to $29.62^{\circ}$ ), 334 (corresponding to $30.62^{\circ}$ ) and 235 (corresponding to $32.43^{\circ}$ ).

The morphology of ZIF-67 (Co) was scanned using SEM. At 10000 times magnification (Figure S3A), it could be clearly seen that a large number of ZIF-67 (CO) particles were closely packed together. At a greater magnification, the shape of ZIF-67 (Co) is similar to a rhombic dodecahedron or a 'chamfered cube' (Figure S3B-C). It was seen that the morphology of ZIF-67 can be changed by adjusting the molar ratio of ligand to metal ion, and the synthesis time. The rhombic dodecahedron grew when the molar ratio of metal ion to ligand was low (Cravillon et al. 2012). In this study, 1:45 was the molar ratio of metal ion to ligand used to form the rhombic dodecahedron (Figure S3D). This resulted in a favorable formation of ZIF-67 (Co) crystal. Additionally, the choice of metal ion salt could directly affect the size of ZIF (Shao et 
al. 2014). When cobalt nitrate was used as the source of cobalt, the ZIF-67 (Co) obtained in our work was an average size of approximately $0.5 \mu \mathrm{m}$.

The functional groups on the surface of ZIF-67 (Co) were analyzed using infrared spectroscopy (Figure S4). 2-methylimidazole, a ligand found exclusively in the ZIF-67 (Co) structure, was used as a standard comparison because it provided most of the infrared absorption signal of ZIF-67 (Co) (Lashgari et al. 2020). The infrared absorption within the range of $1500-1600 \mathrm{~cm}^{-1}$ was attributable to the stretching vibration and bending vibration of the imidazole ring. The peak at $1584 \mathrm{~cm}^{-1}$ corresponded to the stretching vibration of the $\mathrm{C}=\mathrm{N}$ bond in 2-methylimidazole. Lastly, the infrared absorption within the range of $3200-2900 \mathrm{~cm}^{-1}$ belonged to the stretching vibration of $\mathrm{C}-\mathrm{H}$ on the imidazole ring and the aliphatic chain (Lin \&Chang 2015).

\subsection{Characterization of Co-NPCs}

As shown in Figure S5, the XRD characterization was used to obtain the diffraction signal of the synthesized Co-NPC-800 and Co-NPC-800 (Ar). Compared to the XRD spectrum of the original ZIF-67 (Co), a lower resolution and wider particle peaks were observed after carbonization. Meanwhile, there were three obvious diffraction peaks found in both Co-NPC-800 and Co-NPC-800 (Ar), which corresponded well to the elementary substance of $\beta$-Co. Among them, $2 \theta=43.9^{\circ}, 51.1^{\circ}$ and $75.4^{\circ}$ were assigned to (111), (200) and (220) of the crystal plane's diffraction, respectively. In the XRD spectra of both samples, a relatively weak diffraction peak was found at $2 \theta=25^{\circ}$, which belonged to the characteristic peak of the typical (002) graphite laminated structure. This result suggested that the prepared Co-NPXs were partly graphitized. In addition, we observed a faint protrusion at $2 \theta=37^{\circ}$ belonging to the characteristic peak of $\mathrm{C}_{0} \mathrm{O}$. This may be caused by the easy oxidation, in air, of Co nanoparticles on the surface of the porous carbon materials (Ma et al. 2016).

The SEM images of Co-NPC-800 and Co-NPC-800 (Ar) are shown in Figure 2. After carbonization, the original shape of the ZIF-67 (Co) crystal remained but the surfaces of the porous carbon particles were distorted and the size of the particles were shrunk. As shown in Figure 2A-B, the surface of Co-NPC-800 (Ar) was obviously roughened, while the crystals were more concentrated. Compared to Co-NPC-800 (Ar), the surface of Co-NPC-800 was smoother, and the crystals were more dispersed. This result was attributed to the fact that defects were formed on the surface of Co-NPC-800 by nitrogen doping, which separated the crystals and made the dispersive crystals difficult to agglomerate. Additionally, more adsorption sites were available after the defects formed and a larger specific surface area was created after the crystals separated. This increased the chance of Co-NPC-800 contacting with pollutants and caused the adsorption performance to improve.

In order to investigate the changes in the functional groups of ZIF-67 (Co) after carbonization, the infrared spectra of Co-NPC-800 (Ar) and Co-NPC-800 were analyzed (Figure S6). It was found that the infrared spectra of Co-NPC-800 (Ar) and Co-NPC-800 were similar, indicating that their structures were basically the same. Compared to ZIF-67 (Co), Co-NPC retained the normal absorption rate of the aromatic ring in the range of $1500-600 \mathrm{~cm}^{-1}$, which was a result of the fact the imidazole ring remained stable after high 
temperature carbonization. The characteristic peak at $1500 \mathrm{~cm}^{-1}$ corresponded to $\mathrm{C}=\mathrm{C}$ on the aromatic ring, and the peaks at $1300-700 \mathrm{~cm}^{-1}$ were the flexural vibration signals of $\mathrm{C}-\mathrm{H}$ on the aromatic ring. Absorption peaks around $3000 \mathrm{~cm}^{-1}$ were observed in both Co-NPC-800 (Ar) and Co-NPC-800, which may have been caused by the reaction of $\mathrm{C}$ and $\mathrm{H}$ at the edge of the material during pyrolysis. The broad absorption peaks near $3500 \mathrm{~cm}^{-1}$ indicated the stretching vibration of $\mathrm{N}-\mathrm{H}$ or $\mathrm{O}-\mathrm{H}$. The signal of $\mathrm{O}-\mathrm{H}$ may have been a result of the moisture absorbed by the material from the air. Nevertheless, the N-H peak of Co-NPC-800 was sharper than the peak of Co-NPC-800 (Ar), indicating that more $\mathrm{N}$ elements had been successfully introduced into Co-NPC-800 by carbonization in a $\mathrm{N}_{2}$ atmosphere(Ma et al. 2016).

In order to explain the graphitized carbon signal in the XRD spectrum of Co-NPC, Raman spectroscopy was used to further investigate the $C$ element in Co-NPC (Yang et al. 2021). The relative ratio of the Dband area to the G-band area (ID/IG) in the Raman spectrum is an important parameter to understand the degree of crystallization of the graphitized carbon in the materials. The D-band is a region that characteristically contains structural defects and impurities, which represent the defects and disorder of the materials. The G-band is generated by the stretching vibration of $\mathrm{C}-\mathrm{C}$, which represents the internal vibration of $\mathrm{SP}^{2}$ hybrid carbon atoms. The amorphous degree can be expressed by ID/IG, for example, a larger ratio represents a lower degree of order in the graphite materials. The Raman spectra of Co-NPC800 are shown in Figure S7, and ID/IG=2.34 was obtained by fitting. The results indicate that Co-NPC-800 has a low degree pf graphitization, low order and many defects, which could provide more sites for the adsorption of pollutants.

The XPS was used to investigate the composition and element valence of Co-NPC-800, and to further confirm the successful doping of the N element. In the full spectrum (Figure S8A), we could clearly observe the signals of Co, C, N and O. By fitting the N1s spectrum (Figure S8B), we discovered that it could be divided into four independent peaks and that their binding energies were 398.6, 399.3, 400.7 and $404.1 \mathrm{eV}$ respectively. The results suggested that $\mathrm{N}$ elements in Co-NPC-800 existed in four different forms; namely pyridine, pyrrole, graphite and oxidized type. By fitting the N1s spectrum (Figure S8B), it was found that Co element existed in the forms of $\mathrm{Co}(0)$ (at $778.3 \mathrm{eV}$ ), $\mathrm{Co}^{2+}$ (at $780.8 \mathrm{eV}$ ) and $\mathrm{Co}-\mathrm{OH}$ (at $785.7 \mathrm{eV}$ ). This result was consistent with the previous XRD results and results contained in the literature(Huang et al. 2015). The existence of the oxidation state $\mathrm{Co}\left(\mathrm{Co}^{2+}\right)$ verified the partial surface oxidation of Co nanoparticles in the XRD results. The results above showed that high temperature carbonization of ZIF-67 (Co) led to the formation of N-doped magnetic porous carbon materials (Co-NPC), whose magnetic function mainly came from the Co nanoparticles.

The specific surface area, pore type, and adsorption capacity of Co-NPCs were investigated using a $\mathrm{N}_{2}$ adsorption-desorption curve (Fan et al. 2020). $\mathrm{N}_{2}$ adsorption-desorption analysis of Co-NPC-600, Co-NPC800 and Co-NPC-800 (Ar) were carried out at $77 \mathrm{~K}$. The specific surface areas of all three materials exceeded $200 \mathrm{~m}^{2} \cdot \mathrm{g}^{-1}$. Obvious $\mathrm{H} 3$ mesoporous hysteresis loops in the $\mathrm{N}_{2}$ adsorption-desorption curves (Figure S9A), indicated that mesopores formed on ZIF-67 (Co) during the carbonization. The pore size distribution of all three materials were mainly below $10 \mathrm{~nm}$ (Figure S9B), and the average pore sizes were 
all less than $10 \mathrm{~nm}$ (Table 1); this confirmed that Co-NPCs were mesoporous materials. Comparing CoNPC-600 with Co-NPC-800, it was found that the pore size, specific surface area and total pore volume all increased alongside the increase in the carbonization temperature. This implied that the higher temperature led to the greater carbonization of ZIF-67 (Co) and more defects on Co-NPCs. Comparing CoNPC-800 with Co-NPC-800 (Ar), it was found that the pore size, specific surface area and total pore volume of Co-NPC-800 (Ar) were all smaller than those of Co-NPC-800, which showed that Ar was nonreactive with porous carbon materials. Fewer defects and lower parameters were obtained in Ar when compared to $\mathrm{N}_{2}$. Therefore, we concluded that Co-NPC-800 had greater potential in adsorption applications.

Table 1

Structural parameters fitted by $\mathrm{N}_{2}$ adsorption model at $77 \mathrm{~K}$

\begin{tabular}{|lllll|}
\hline Entry & Sample & Aperture $(\mathrm{nm})$ & $S_{B E T}\left(\mathrm{~m}^{2} \cdot \mathrm{g}^{-1}\right)$ & Pore volume $\left(\mathrm{cm}^{3} \cdot \mathrm{g}^{-1}\right)$ \\
\hline 1 & Co-NPC-600 & 6.1517 & 240.8376 & 0.318171 \\
\hline 2 & Co-NPC-800 & 9.9545 & 275.5626 & 0.389612 \\
\hline 3 & Co-NPC-800 (Ar) & 9.6441 & 259.8515 & 0.3662 \\
\hline
\end{tabular}

As shown in Figure 3, the magnetization curves of Co-NPCs were obtained in the magnetic field range of $\pm 20,000$ Oe, at room temperature. It can be seen that four kinds of Co-NPC each had small hysteresis loops. The magnetization of the Co-NPCs rose slowly when the magnetic field exceeded $\pm 10,000$ Oe, which explained why Co-NPCs were soft magnetic materials. As the temperature increased, the saturation magnetization (Ms) of Co-NPC rose from 35.05 to $65.25 \mathrm{emu} \cdot \mathrm{g}^{-1}$. The increase of $M s$ was due to the higher crystallinity of Co nanoparticles embedded in Co-NPCs as the temperature rose (Torad et al. 2014). The high Ms value of Co-NPC-800 came from the large load of Co nanoparticles. Similar studies of magnetic porous carbon materials have also been reported (Hameed 2009, Hameed \&Lee 2009, Ji et al. 2009, Ravikumar et al. 2005, Wu et al. 2012), however, the Ms value of Co-NPC-800 was higher than them. As shown in the magnetic separation photo, Co-NPC-800 could be quickly separated by an external magnetic field after being dispersed in water.

\subsection{Investigation into the factors affecting the adsorption of TCS and PCMX by Co-NPCs}

\subsubsection{Effect of carbonization temperature and atmosphere}

Temperature can affect the carbonization degree of materials (Guo et al. 2017), while different atmospheres affect the surface reaction during the materials' carbonization (Ismail et al. 2018). The adsorption capacity of synthesized Co-NPCs within different atmospheres and under different temperatures for TCS and PCMX had been compared (Figure 4). It can be seen that the influence of temperature and atmosphere on the adsorption of TCS and PCMX were almost the same, which related to 
the same charge properties of both under the experimental conditions $(\mathrm{pH}=7.0)$. As the temperature rose, the adsorption capacity of Co-NPC-600, Co-NPC-700 and Co-NPC-800 for TCS and PCMX gradually increased, which mainly because the pore size, specific surface area and total pore volume of Co-NPC all increased with the carbonization temperature. The contact area with pollutants and volume containing pollutants was also increased. Compared with Co-NPC-800 (Ar), the adsorption capacity of the Co-NPC800 was significantly enhanced. Synthesized in a $\mathrm{N}_{2}$ atmosphere, the Co-NPC-800 doping of $\mathrm{N}$ obtained more adsorption sites, which was conducive to the adsorption of TCS and PCMX. Out of all the materials, Co-NPC-800 was selected for follow-up experiments because it had the best adsorption capacity.

\subsubsection{Effect of pH}

The $\mathrm{pH}$ of the solution can affect the existing form of the adsorbate in the solution, and it could also affect the charge properties on the adsorbent's surface (Bernal et al. 2017). The effect of the pH on the adsorption of TCS and PCMX by Co-NPC-800 was investigated. With the increase of $\mathrm{pH}$, it could be seen that the adsorption capacity of Co-NPC-800 for TCS and PCMX both increased firstly and then decreased (Figure 5A\&C). The results implied that electrostatic attraction might be the main mechanism Co-NPC-800 for the adsorption of pollutants. In order to verify this conjecture, the existing forms of TCS and PCMX were analyzed at different pH levels (Figure 5B\&D). The pKa of TCS and PCMX were 7.68 and 9.21, respectively. The TCS was mainly in form of neutral molecules in the solution when the $\mathrm{pH}$ was less than 7.68. Most of the molecules would carry one unit of negative charge after losing a proton where the $\mathrm{pH}$ was greater than 7.68. The PCMX was mainly in form of neutral molecules in the solution when the pH was less than 9.21, and most of molecules would carry one unit of negative charge after losing a proton, when the $\mathrm{pH}$ was greater than 9.21 .

At the same time, the Zeta potential of Co-NPC-800 was investigated when the $\mathrm{pH}$ was between 4.0-9.0 (Figure S10). It could be seen that the Co-NPC-800 was always negative in the whole range, and the negative charge showed a slight downward trend when the $\mathrm{pH}$ increased between the range of 4.0-7.0. The negative charge showed an obvious up-trend when the $\mathrm{pH}$ increased through the range of 7.0-9.0. Considering the form of the pollutants and the concept of Zeta potential, if there was only an electrostatic effect impacting upon the process, the optimal adsorption pH of Co-NPC-800 for TCS and PCMX should be around 7.0. At this time, Co-NPC-800 had the lowest negative charge and the pollutants were not charged, which are the most beneficial conditions for an electrostatic effect. However, whilst the experimental results showed that best adsorption $\mathrm{pH}$ of Co-NPC-800 for PCMX was around 7.0, the best adsorption $\mathrm{pH}$ of Co-NPC-800 for TCS was around 6.0. It could be assumed that electrostatic effect was not the only mode of action in the adsorption of Co-NPC-800 to TCS, but also there were $\pi-\pi$ and hydrogen bond effects. Under acidic conditions, the $\pi-\pi$ effect appeared between the imidazole ring on the surface of Co-NPC-800 and the benzene ring of the TCS molecule, which benzene group could be used as a $\pi$ donor or acceptor. What's more, the hydroxyl group of TCS molecules could be used as an $\mathrm{H}$ donor to form a hydrogen bond with the $\mathrm{N}$ atom on the surface of Co-NPC-800. The chlorine/hydroxyl group of TCS molecules could also be used as an $\mathrm{H}$ receptor to form a hydrogen bond with the $\mathrm{H}$ atom of Co-NPC-800. In conclusion, the optimal adsorption pH of TCS was 6.0 due to the multiple factors above. 
Because of the large steric hindrance caused by two methyl groups on the surface of PCMX, $\pi-\pi$ and hydrogen bond effects were in the minority during PCMX adsorption. As a result, the $\mathrm{pH}$ of PCMX adsorption remained at 7.0.

\subsubsection{Effect of ionic strength}

A high concentration of salt in industrial waste water might affect the removal of pollutants by Co-NPC. So, the effect of solution ionic strength on the adsorption of TCS and PCMX was we studied by Co-NPC800. As shown in Figure S11A, the ionic strength had only had a small impact on TCS adsorption. When the $\mathrm{NaCl}$ concentration was above $0.2 \mathrm{molY}^{-1}$, the adsorption capacity increased slightly as the $\mathrm{NaCl}$ concentration rose. According to the existing form of TCS and the surface charge of Co-NPC-800, the $\mathrm{Na}^{+}$ between the Co-NPC-800 and TCS molecules would generate a shielding effect, which was beneficial to the interaction between adsorbate and adsorbent, and enhanced the adsorption of Co-NPC-800 to TCS. On the contrary, the adsorption capacity of Co-NPC-800 for PCMX became weaker and tended to be stable when more $\mathrm{NaCl}$ was added (Figure S11B). This result might be attributed to the fact that the adsorption of Co-NPC-800 for PCMX had, for the most part, an electrostatic effect rather than $\pi-\pi$ effect, while $\mathrm{NaCl}$ would compete with PCMX for the adsorption sites on Co-NPC-800. Therefore, the binding sites of PCMX and Co-NPC-800 were partially occupied by $\mathrm{NaCl}$, resulting in a decrease in its adsorption capacity. Nevertheless, under experimental conditions, the salt ion concentration had a very little effect on the adsorption of TCS and PCMX by Co-NPC-800.

\subsection{Study on the Adsorption Kinetics of Co-NPC-800 on TCS and PCMX}

By investigating the change of absorbance between Co-NPC-800, TCS and PCMX, the time taken to reach adsorption equilibrium could be determined. In addition, the adsorption mechanism could be better understood by fitting the kinetic model. As shown in Figure 6A\&D, the equilibrium time of adsorption on Co-NPC-800 $\left(0.1 \mathrm{mg} \cdot \mathrm{mL}^{-1}\right)$ with TCS $\left(20 \mathrm{mg} \cdot \mathrm{L}^{-1}\right)$ and PCMX $\left(10 \mathrm{mg} \cdot \mathrm{L}^{-1}\right)$ were both $3 \mathrm{mins}$. Although both TCS and PCMX were highly concentrated, Co-NPC-800 showed excellent adsorptive power for these two targets.

Fitting the experimental data with the quasi-first-order and the quasi-second-order kinetic equation respectively, the fitting curves obtained are showing in Figure 6B-C\&E-F, while the kinetic parameters and correlation coefficients for TCS and PCMX adsorbed by Co-NPC-800 are summarized in Table S1. It can be seen that the adsorption kinetics of Co-NPC-800 on TCS and PCMX were more in line with quasisecond-order kinetic model $\left(R^{2}>0.99\right)$, the adsorption capacity calculated by the quasi-second-order kinetic equation were closer to the experimental value as well. Above results suggested that the quasisecond-order kinetic model could be applied to the adsorption process of TCS and PCMX.

\subsection{Adsorption isotherms of TCS and PCMX}

The adsorption isotherms of TCS and PCMX by Co-NPC-800 were studied at 298,308 , and $318 \mathrm{~K}$. The concentration range of TCS and PCMX were $8-40$ and $1-25 \mathrm{mg} \cdot \mathrm{L}^{-1}$, respectively. As the concentration of 
TCS and PCMX increased, the adsorption capacity increased rapidly and tended to balance. Under experimental conditions, the adsorption capacity of Co-NPC-800 for TCS and PCMX also increased as the temperature rose (Figure 7A\&D). In line with the Langmuir adsorption isotherm equation and Freundlich adsorption isotherm equation, it was possible to obtain fitted linear diagrams (Figure 7B-C\&E-F). The related parameters are shown in Table $\mathbf{S 2}$.

Only single-layer with physical adsorption was assumed in the Langmuir model. However, in the Freudlich model, single-layer and multi-layer adsorption were combined and both chemical and physical adsorption was considered. According to the fitted results, it could be seen that the adsorption of TCS and PCMX by Co-NPC-800 were more in line with the Langmuir isotherm adsorption model $\left(R^{2}>0.99\right)$, while the Freundlich model fit relatively poorly. The results suggested that the type of adsorption of TCS and PCMX by Co-NPC-800 was physical adsorption with a single-layer covering, which was also in line with the results of kinetics $\left(q_{m} \approx q_{2 e}\right)$. As the temperature increased, the adsorption constant of the Langmuir model $\left(K_{L}\right)$ gradually increased. The maximum adsorption capacity $\left(q_{m}\right)$ of TCS increased from 163 to $263 \mathrm{mg} \cdot \mathrm{g}^{-1}$, and the maximum adsorption capacity of PCMX increased from 39 to $56 \mathrm{mg} \cdot \mathrm{g}^{-1}$. These results show that the adsorption of TCS and PCMX by Co-NPC-800 is an endothermic process.

Additionally, the adsorption constant of the Freundlich model $\left(K_{F}\right)$ also increased as the temperature rose, and the value of $n$ was greater than 1 at all the temperature, indicating that the adsorption of both pollutants by Co-NPC-800 was a preferential adsorption process.

\subsection{Study on the adsorption thermodynamics of TCS and PCMX}

In order to further understand the adsorption mechanism of TCS and PCMX on Co-NPC-800, we calculated thermodynamic parameters including Gibbs free energy change $\left(\Delta G^{9}\right)$, enthalpy change $\left(\Delta H^{9}\right)$ and entropy change $\left(\Delta S^{9}\right)$. The $\Delta G^{0}$ value indicated the priority in the adsorption process and the magnitude of the adsorption driving force. The adsorption process could not be carried out spontaneously when $\Delta G^{0}>0$, but the adsorption process could be carried out spontaneously when $\Delta G^{0}<0$ (Tran et al. 2020). As listed in Table 2, the $\Delta G^{0}$ of Co-NPC-800 adsorbing TCS and PCMX were both negative in the range of $298-318 \mathrm{~K}$. This indicated that that both processes could proceed spontaneously. Meanwhile, the $\Delta G^{0}$ decreased as the temperature rose, suggesting that the temperature rise enhanced the driving force of adsorption and promoted the adsorption progress. This result also inferred that the adsorption of TCS and PCMX on Co-NPC-800 was an endothermic process. Further, the $\Delta H^{0}>0$ also proved that the two processes were endothermic. The results calculated for $\Delta G^{0}$ and $\Delta H^{0}$ confirmed the results of adsorption isotherm. In general, having $\Delta G^{0}$ between -80 and $400 \mathrm{~kJ} \cdot \mathrm{mol}^{-1}$ was considered to be chemical adsorption, while $\Delta G^{0}$ between -20 and $0 \mathrm{~kJ} \cdot \mathrm{mol}^{-1}$ was regarded as physical adsorption (Özcan et al. 2005). In this study, the $\Delta G^{0}$ values of the two adsorption processes were in the range of -20$0 \mathrm{~kJ} \cdot \mathrm{mol}^{-1}$, suggesting that both of them were physical adsorption. These results were also in line with the results of kinetics and adsorption isotherms. 
Table 2

Thermodynamic parameters of adsorption on Co-NPC-800 to TCS and PCMX

\begin{tabular}{|llllll|}
\hline Target & Temperature $(\mathrm{K})$ & $K$ & $\Delta G^{\circ}\left(\mathrm{kJ} \cdot \mathrm{mol}^{-1}\right)$ & $\Delta H^{\circ}\left(\mathrm{kJ} \cdot \mathrm{mol}^{-1}\right)$ & $\Delta S^{\circ}\left(\mathrm{kJ} \cdot \mathrm{mol}^{-1} \cdot \mathrm{K}^{-1}\right)$ \\
\hline TCS & 298 & 21.558 & -7.608 & 27.843 & -0.119 \\
\cline { 2 - 6 } & 308 & 29.282 & -8.647 & & \\
\cline { 2 - 6 } & 318 & 40.93 & -9.814 & & \\
\hline PCMX & 298 & 19.057 & -7.302 & 9.019 & -0.0547 \\
\cline { 2 - 6 } & 308 & 20.737 & -7.512 & & \\
\hline
\end{tabular}

The $\Delta S^{0}$ value reflects a change in system disorder. It was a process of entropy increase when $\Delta S^{0}>0$, while it was a process of entropy decrease when $\Delta S^{0}<0$. The $\Delta S^{0}$ was below 0 in the adsorption process of Co-NPC-800 on TCS and PCMX, indicating that, after adsorption, the disorder of the system was reduced.

The massive desorption of water molecules arranged on the surface of Co-NPC-800 would increase the disorder of the entire system and increase the entropy in the adsorption process. However, when TCS and PCMX were adsorbed from the solution to the solid-liquid interface of the adsorbent, some translational and rotational degrees of freedom were lost, which led to a decrease in entropy. The entropy decrease in this process was greater than the entropy increase caused by the massive desorption of water molecules. Therefore, overall, the entire adsorption process showed a decrease in entropy.

\subsection{Adsorption mechanism}

As mentioned above, the adsorption of Co-NPC-800 to TCS and PCMX was mainly physical adsorption, which included electrostatic, $\pi-\pi$ and hydrogen bond effects. Electrostatic interaction is one of the most common. In order to further verify the role of $\pi-\pi$ and the hydrogen bond, an infrared spectrometer was used to investigate Co-NPC-800 after adsorption of TCS and PCMX. As shown in Figure 8A\&B, obvious infrared absorptions at $1500-700 \mathrm{~cm}^{-1}, 3000 \mathrm{~cm}^{-1}$ and $3500 \mathrm{~cm}^{-1}$ were observed. Compared with the spectra of pure TCS and PCMX, the characteristic peaks were weaker, despite the fact it implied that TCS and PCMX were successfully adsorbed on Co-NPC-800. The slight shift of the characteristic hydroxyl peak at $3500 \mathrm{~cm}^{-1}$ was attributed to the formation of a hydrogen bond, which is between the $\mathrm{N}$ atom on the surface of Co-NPC-800 and the hydroxyl groups in the TCS/PCMX molecules. The characteristic peak of the $\mathrm{C}=\mathrm{C}$ skeleton vibration in the aromatic ring (about $1600 \mathrm{~cm}^{-1}$ ) also shifted slightly, and became wider after adsorption. This could be attributed to the $\pi-\pi$ effect between TCS/PCMX molecules and the imidazole ring that remained after carbonization. Due to the large steric hindrance in the PCMX molecule caused by multiple substituents on the benzene ring, the $\pi-\pi$ effect between Co-NPC-800 and PCMX was suppressed. So, the adsorption capacity of Co-NPC-800 for PCMX was inferior to that of TCS, which meant the steric hindrance was lesser. 
In addition, the hydrogen bond acceptors and donors of TCS and PCMX at different pH were analyzed, and the results are shown in Figure 8C\&D. There were one hydrogen bond acceptor and two hydrogen bond donors in TCS when $\mathrm{pH}<6.0$. This was consistent with the best adsorption capacity of Co-NPC-800 to TCS at $\mathrm{pH}=6$. The results confirmed that the adsorption of Co-NPC-800 to TCS had several modes of action. For example, there was one hydrogen bond acceptor and two hydrogen bond donors in PCMX when $\mathrm{pH}<7.0$. These were consistent with the best adsorption capacity of Co-NPC-800 to PCMX at $\mathrm{pH}=7.0$. The results indicated that the hydrogen bond was also important in the adsorption of Co-NPC800 to PCMX. The mechanism diagram of the adsorption on Co-NPC-800 to TCS and PCMX was drawn according to above-mentioned experimental and theoretical results (Figure 9). Co-NPC-800 was also highly able to remove TCS and PCMX due to the combined of electrostatic, $\pi-\pi$ and hydrogen bond effects.

\subsection{Investigation on the reusability}

As shown in Figure S12, the adsorption capacity of Co-NPC-800 for TCS remained above $150 \mathrm{mg} \cdot \mathrm{g}^{-1}$ even after being recycled 5 times, showing that Co-NPC-800 has good reusability. However, the adsorption capacity of Co-NPC-800 for PCMX decreased from 40 to $25 \mathrm{mg} \cdot \mathrm{g}^{-1}$ after being recycled 5 times. This could be explained by the fact that the adsorption capacity of Co-NPC-800 for PCMX was originally small, and the sites where Co-NPC-800 adsorbed PCMX were partially deactivated during the cycles.

\subsection{Application of Co-NPC in actual water samples}

In order to verify the ability to practically apply this method in this work, we investigated the removal performance of Co-NPC-800 for TCS and PCMX in actual water samples. Both TCS and PCMX were not detected in the collected lake water, river water and tap water, so TCS with spiked concentration of 20 $\mathrm{mg} \cdot \mathrm{L}^{-1}$ and PCMX with spiked concentration of $10 \mathrm{mg} \cdot \mathrm{L}^{-1}$ were added in the three water samples. The results of pollutant absorption are shown in Figure 10. The maximum adsorption capacity of Co-NPC-800 for TCS and PCMX in the three water samples was close to the theoretical values, which were about 160 and $40 \mathrm{mg} \cdot \mathrm{L}^{-1}$. Co-NPC-800 could be used as an adsorbent for the removal of TCS and PCMX in sewage, and it has a great potential to be applied more broadly.

\section{Conclusion}

In this work, ZIF-67 (Co), a kind of MOF material with a zeolite structure, was synthesized using the 'onepot method'. Co-NPC, the porous carbon material mixed with nitrogen element, was prepared causing ZIF67 (Co) carbonization at high temperatures within an atmosphere containing $\mathrm{N}_{2}$. The obtained Co-NPC, retaining the original structure of ZIF-67 (Co) crystals, had good porosity, magnetism and large specific surface area. The cobalt element in the ZIF-67 (Co) framework remained in the material inside the form of cobalt oxide, wherein provided magnetism to the material. The Co-NPC-800 had good adsorption capacity for TCS and PCMX in water. The adsorption of Co-NPC-800 on both reached equilibrium within 3 minutes, and was in accord with the quasi-second-order kinetic model. At $298 \mathrm{~K}$, the maximum adsorption capacity of Co-NPC-800 for TCS and PCMX was 163 and $39 \mathrm{mg} \cdot \mathrm{g}^{-1}$, respectively. The adsorption was in line with 
the Langmuir isotherm adsorption model. Moreover, the adsorption of TCS and PCMX by Co-NPC-800 was a spontaneous endothermic process with reduced entropy. The excellent adsorption capacity of CoNPC-800 is a result of multiple properties including electrostaticity, $\pi-\pi$ and hydrogen bond effects. Moreover, Co-NPC-800 can be regenerated through simple washing and can be reused several times. CoNPC-800 is a magnetic and recyclable adsorbent with high adsorption capacity and stability, thus is valuable and may be broadly used in sewage treatment and other environmental fields.

\section{Declarations}

\section{Acknowledgements}

The authors gratefully acknowledge the Sichuan Science and Technology Program (No. 19JCQN0061).

\section{Author information}

\section{Affiliations}

College of Ecology and Environment, Chengdu University of Technology, Chengdu 610059, China

\section{Qiuxing Li}

College of Earth Science, Chengdu University of Technology, Chengdu 610059, China

\section{Paijin Zhu}

State Environmental Protection Key Laboratory of Synergetic Control and Joint Remediation for Soil \& Water Pollution, Chengdu University of Technology, Chengdu 610059, China

\section{Paijin Zhu, Li Huang, Min Zhong, Shuxia Xu}

College of Ecology and Environment, Chengdu University of Technology, Chengdu, Sichuan 610059, P. R. China

Corresponding author

Correspondence to Shuxia Xu

\section{Shuxia Xu}

Corresponding author

\section{Contributions}

Qiuxing Li contributed to the interpretation of the results and to the writing of the manuscript. Paijin Zhu collected the samples and carried the experiment. Li Huang collected the samples. Min Zhong revised the manuscript. Shuxia Xu supervised the study. All the authors read and approved the final manuscript. 
Availability of data and materials

All data generated or analyzed during the current study are included in this article and its supplementary information files.

\section{Ethics declarations}

\section{Ethics approval}

Not applicable.

\section{Consent to participate}

Not applicable.

\section{Consent for publication}

Yes, approval of all authors.

\section{Competing interest}

The authors declare that they have no competing interest.

\section{References}

1. Ahmaruzzaman M (2008) Adsorption of phenolic compounds on low-cost adsorbents: a review.Advances in colloid and interface science143:48-67

2. Apul OG, Wang Q, Zhou Y, Karanfil T (2013) Adsorption of aromatic organic contaminants by graphene nanosheets: Comparison with carbon nanotubes and activated carbon.Water research47:1648-1654

3. Banerjee R, Phan A, Wang B, Knobler C, Furukawa H, O'Keeffe M, Yaghi OM (2008) High-throughput synthesis of zeolitic imidazolate frameworks and application to CO2 capture.Science319:939-943

4. Bao C, Fang C-I (2012) Water resources flows related to urbanization in China: challenges and perspectives for water management and urban development. Water resources management26:531552

5. Bernal V, Erto A, Giraldo L, Moreno-Piraján JC (2017) Effect of solution pH on the adsorption of paracetamol on chemically modified activated carbons.Molecules22:1032

6. Bhadra BN, Lee JK, Cho C-W, Jhung SH (2018) Remarkably efficient adsorbent for the removal of bisphenol A from water: Bio-MOF-1-derived porous carbon.Chemical Engineering Journal343:225234

7. Chaikittisilp W, Ariga K, Yamauchi Y (2013) A new family of carbon materials: synthesis of MOFderived nanoporous carbons and their promising applications.Journal of Materials Chemistry 


\section{A1:14-19}

8. Chen W, Pei J, He CT, Wan J, Ren H, Wang Y, Dong J, Wu K, Cheong WC, Mao J (2018) Single tungsten atoms supported on MOF-derived N-doped carbon for robust electrochemical hydrogen evolution.Advanced Materials30:1800396

9. Cravillon J, Schröder CA, Bux H, Rothkirch A, Caro J, Wiebcke M (2012) Formate modulated solvothermal synthesis of ZIF-8 investigated using time-resolved in situ X-ray diffraction and scanning electron microscopy.CrystEngComm14:492-498

10. Crini G, Lichtfouse E, Wilson LD, Morin-Crini N (2019) Conventional and non-conventional adsorbents for wastewater treatment.Environmental Chemistry Letters17:195-213

11. Diaconu I, Gîrdea R, Cristea C, Nechifor G, Ruse E, Totu EE (2010) Removal and recovery of some phenolic pollutants using liquid membranes.Romanian Biotechnological Letters15:5702-5708

12. Fan Y, Li S, Wang Y, Zhuang C, Liu X, Zhu G, Zou X (2020) Tuning the synthesis of polymetallic-doped ZIF derived materials for efficient hydrogenation of furfural to furfuryl alcohol.Nanoscale12:1829618304

13. Guo Y, Tang J, Salunkhe RR, Alothman ZA, Hossain MSA, Malgras V, Yamauchi Y (2017) Effect of various carbonization temperatures on ZIF-67 derived nanoporous carbons.Bulletin of the Chemical Society of Japan90:939-942

14. Gupta VK, Atar N, Yola ML, Üstündağ Z, Uzun L (2014) A novel magnetic Fe@ Au core-shell nanoparticles anchored graphene oxide recyclable nanocatalyst for the reduction of nitrophenol compounds. Water research48:210-217

15. Hameed B (2009) Evaluation of papaya seeds as a novel non-conventional low-cost adsorbent for removal of methylene blue.Journal of Hazardous materials162:939-944

16. Hameed B, Lee T (2009) Degradation of malachite green in aqueous solution by Fenton process.Journal of hazardous materials164:468-472

17. Huang J, Tan Y, Su J, Gu Q, Černý R, Ouyang L, Sun D, Yu X, Zhu M (2015) Synthesis, structure and dehydrogenation of zirconium borohydride octaammoniate.Chemical Communications51:27942797

18. Ismail N, Salleh W, Sazali N, Ismail A, Yusof N, Aziz F (2018) Disk supported carbon membrane via spray coating method: Effect of carbonization temperature and atmosphere.Separation and Purification Technology195:295-304

19. Ji B, Shao F, Hu G, Zheng S, Zhang Q, Xu Z (2009) Adsorption of methyl tert-butyl ether (MTBE) from aqueous solution by porous polymeric adsorbents.Journal of hazardous materials161:81-87

20. Kasmi N, Majdoub M, Papageorgiou GZ, Achilias DS, Bikiaris DN (2017) Solid-state polymerization of poly (ethylene furanoate) biobased polyester, I: Effect of catalyst type on molecular weight increase.Polymers9:607

21. Lashgari SM, Yari H, Mahdavian M, Ramezanzadeh B, Bahlakeh G, Ramezanzadeh M (2020) Unique 2-methylimidazole based Inorganic Building Brick nano-particles (NPs) functionalized with 3aminopropyltriethoxysilane with excellent controlled corrosion inhibitors delivery performance; 
Experimental coupled with molecular/DFT-D simulations.Journal of the Taiwan Institute of Chemical Engineers117:209-222

22. Lee J, Han S, Hyeon T (2004) Synthesis of new nanoporous carbon materials using nanostructured silica materials as templates.Journal of Materials Chemistry 14:478-486

23. Liang C, Tang Y, Zhang X, Chai H, Huang Y, Feng P (2020) ZIF-mediated N-doped hollow porous carbon as a high performance adsorbent for tetracycline removal from water with wide $\mathrm{pH}$ range.Environmental research182:109059

24. Lin K-YA, Chang H-A (2015) Ultra-high adsorption capacity of zeolitic imidazole framework-67 (ZIF67) for removal of malachite green from water.Chemosphere139:624-631

25. Liu L, Zhu YP, Su M, Yuan ZY (2015) Metal-free carbonaceous materials as promising heterogeneous catalysts.ChemCatChem7:2765-2787

26. Lou Z, Li R, Liu J, Wang Q, Zhang Y, Li Y (2021) Used dye adsorbent derived N-doped magnetic carbon foam with enhanced electromagnetic wave absorption performance.Journal of Alloys and Compounds854:157286

27. Lv Z-S, Zhu X-Y, Meng H-B, Feng J-J, Wang A-J (2019) One-pot synthesis of highly branched Pt@ Ag core-shell nanoparticles as a recyclable catalyst with dramatically boosting the catalytic performance for 4-nitrophenol reduction.Journal of colloid and interface science538:349-356

28. Ma X, Zhou Y-X, Liu H, Li Y, Jiang H-L (2016) A MOF-derived Co-CoO@ N-doped porous carbon for efficient tandem catalysis: dehydrogenation of ammonia borane and hydrogenation of nitro compounds.Chemical Communications52:7719-7722

29. Mahugo Santana C, Sosa Ferrera Z, Esther Torres Padrón M, Juan Santana Rodríguez J (2009) Methodologies for the extraction of phenolic compounds from environmental samples: New approaches.Molecules14:298-320

30. Martín MB, Pérez JS, Sánchez JG, de Oca LM, López JC, Oller I, Rodríguez SM (2008) Degradation of alachlor and pyrimethanil by combined photo-Fenton and biological oxidation.Journal of hazardous materials155:342-349

31. Meng F, Song M, Chen Y, Wei Y, Song B, Cao Q (2021) Promoting adsorption of organic pollutants via tailoring surface physicochemical properties of biomass-derived carbon-attapulgite.Environmental Science and Pollution Research28:11106-11118

32. Ng A, Weerakoon D, Lim E, Padhye LP (2019) Fate of environmental pollutants. Water Environment Research91:1294-1325

33. Oladipo AS, Ajayi OA, Oladipo AA, Azarmi SL, Nurudeen Y, Atta AY, Ogunyemi SS (2018) Magnetic recyclable eggshell-based mesoporous catalyst for biodiesel production from crude neem oil: Process optimization by central composite design and artificial neural network.Comptes Rendus Chimie21:684-695

34. Oturan N, Panizza M, Oturan MA (2009) Cold incineration of chlorophenols in aqueous solution by advanced electrochemical process electro-Fenton. Effect of number and position of chlorine atoms on the degradation kinetics. The Journal of Physical Chemistry A113:10988-10993 
35. Özcan AS, Erdem B, Özcan A (2005) Adsorption of Acid Blue 193 from aqueous solutions onto BTMA-bentonite.Colloids and Surfaces A: Physicochemical and Engineering Aspects266:73-81

36. Rahmanian N, Jafari SM, Galanakis CM (2014) Recovery and removal of phenolic compounds from olive mill wastewater.Journal of the American Oil Chemists' Society91:1-18

37. Ravikumar K, Deebika B, Balu K (2005) Decolourization of aqueous dye solutions by a novel adsorbent: application of statistical designs and surface plots for the optimization and regression analysis.Journal of hazardous materials122:75-83

38. Shao J, Wan Z, Liu H, Zheng H, Gao T, Shen M, Qu Q, Zheng H (2014) Metal organic frameworksderived $\mathrm{Co} 3 \mathrm{O} 4$ hollow dodecahedrons with controllable interiors as outstanding anodes for $\mathrm{Li}$ storage.Journal of Materials Chemistry A2:12194-12200

39. Sule R, Mishra AK (2020) MOFs-carbon hybrid nanocomposites in environmental protection applications.Environmental Science and Pollution Research27:16004-16018

40. Tan J, Kuang H, Wang C, Liu J, Pang Q, Xie Q, Fan R (2021) Human exposure and health risk assessment of an increasingly used antibacterial alternative in personal care products: Chloroxylenol.Science of The Total Environment786:147524

41. Teimouri Z, Salem A, Salem S (2019) Regeneration of wastewater contaminated by cationic dye by nanoporous activated carbon produced from agriculture waste shells. Environmental Science and Pollution Research26:7718-7729

42. Torad NL, Hu M, Ishihara S, Sukegawa H, Belik AA, Imura M, Ariga K, Sakka Y, Yamauchi Y (2014) Direct synthesis of MOF-derived nanoporous carbon with magnetic Co nanoparticles toward efficient water treatment.Small10:2096-2107

43. Tran HV, Hoang LT, Huynh CD (2020) An investigation on kinetic and thermodynamic parameters of methylene blue adsorption onto graphene-based nanocomposite.Chemical Physics535:110793

44. Vakili F, Rashidi A, Taghavi L, Mansouri N (2021) Single-step synthesis of N, S co-doped wastederived nanoporous carbon sorbent for mercury vapor removal.Environmental Science and Pollution Research28:17265-17274

45. Wada S, Ichikawa H, Tastsumi K (1995) Removal of phenols and aromatic amines from wastewater by a combination treatment with tyrosinase and a coagulant.Biotechnology and Bioengineering 45:304-309

46. Wang C, Yin H, Tian P, Sun X, Pan X, Chen K, Chen W-J, Wu Q-H, Luo S (2020) Remarkable adsorption performance of MOF-199 derived porous carbons for benzene vapor.Environmental research184:109323

47. Wu Z, Li W, Webley PA, Zhao D (2012) General and controllable synthesis of novel mesoporous magnetic iron oxide@ carbon encapsulates for efficient arsenic removal.Advanced Materials24:485491

48. Yang X, Yang W, Fu X, Hu J, Chen J (2021) N-enriched porous carbon doped with co, ni, and Mo as efficient electrocatalyst for hydrogen evolution reaction.International Journal of Hydrogen Energy 46:18318-18325

Page 19/25 
49. Yao Y, Zhang J, Wu G, Wang S, Hu Y, Su C, Xu T (2017) Iron encapsulated in 3D N-doped carbon nanotube/porous carbon hybrid from waste biomass for enhanced oxidative activity. Environmental Science and Pollution Research24:7679-7692

50. Yoo DK, An HJ, Khan NA, Hwang GT, Jhung SH (2018) Record-high adsorption capacities of polyaniline-derived porous carbons for the removal of personal care products from water.Chemical Engineering Journal352:71-78

51. Yu F, Bai X, Liang M, Ma J (2020) Recent progress on metal-organic framework-derived porous carbon and its composite for pollutant adsorption from liquid phase.Chemical Engineering Journal405:126960

52. Zhang W, Huang Y, Zhu S, Li X, Liu J, Zhang Y (2015) Preparation of reed hemicelluloses-based hydrogels and its highly effective adsorption of heavy metal ions.Chemistry and Industry of Forest Products35:28-34

\section{Figures}

\section{Image not available with this version}

\section{Figure 1}

Figure 1 


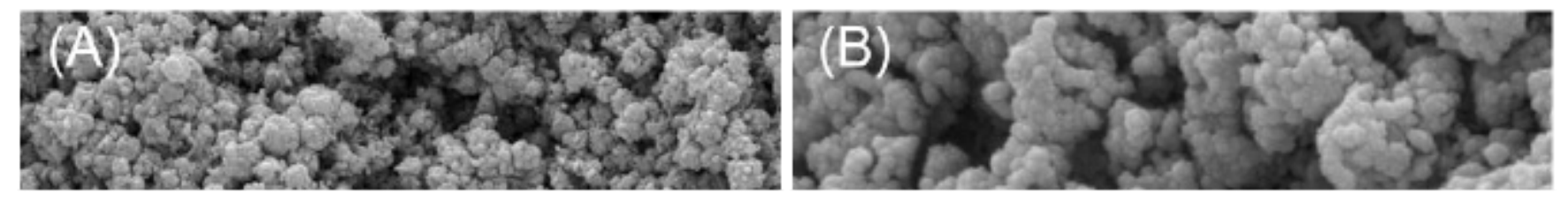

Figure 2

SEM results of synthesized Co-NPC-800 (Ar) at (A) 50,000x and (B) 200,000x magnification and Co-NPC800 at (C) 50,000x and (D) 200,000x magnification. 


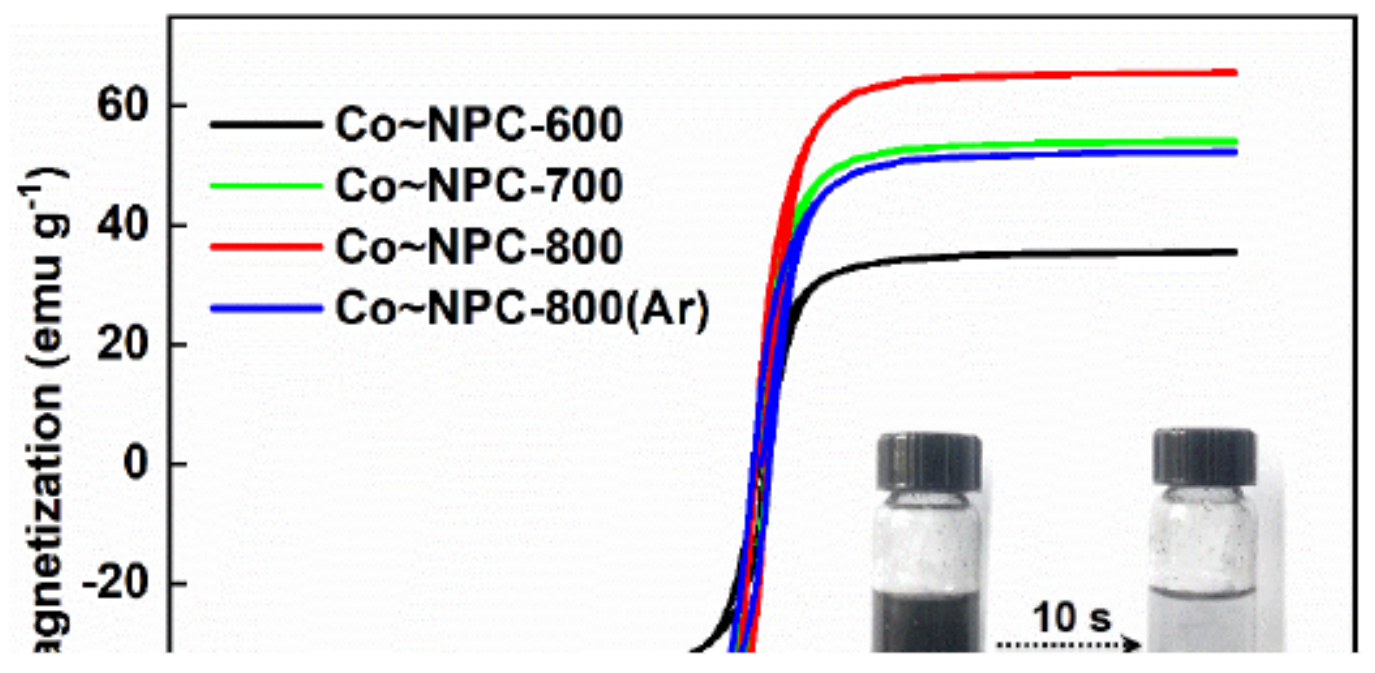

Figure 3

Magnetic hysteresis loop of Co-NPCs and magnetic separation photo of Co-NPC-800.

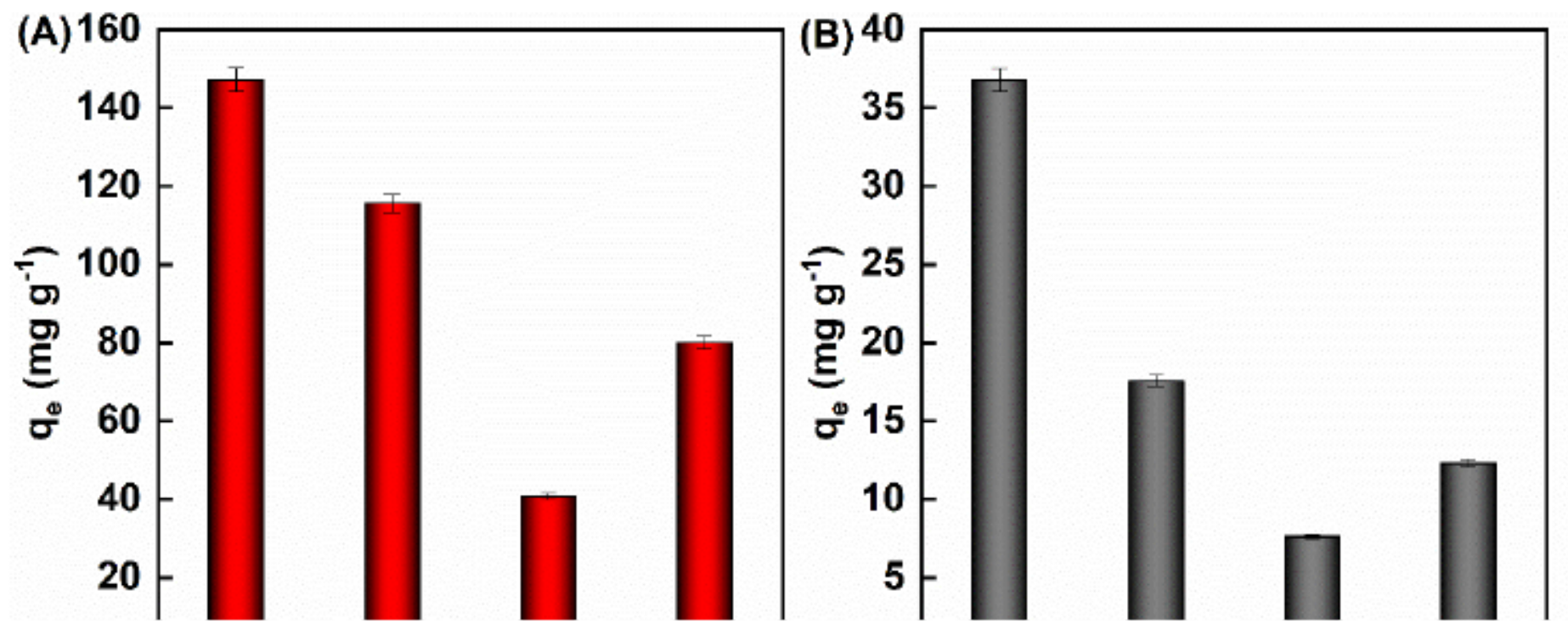

Figure 4 
Comparison of pollutant adsorption capacity by Co-NPC-600, Co-NPC-700, Co-NPC-800 and Co-NPC-800 (Ar). (A) TCS and (B) PCMX.

Figure 5

A variety of properties at different pH. (A) Adsorption capacity of Co-NPC-800 to TCS; (B) Micro-species distribution of TCS; (C) Adsorption capacity of Co-NPC-800 to PCMX区(D) Micro-species distribution of PCMX.
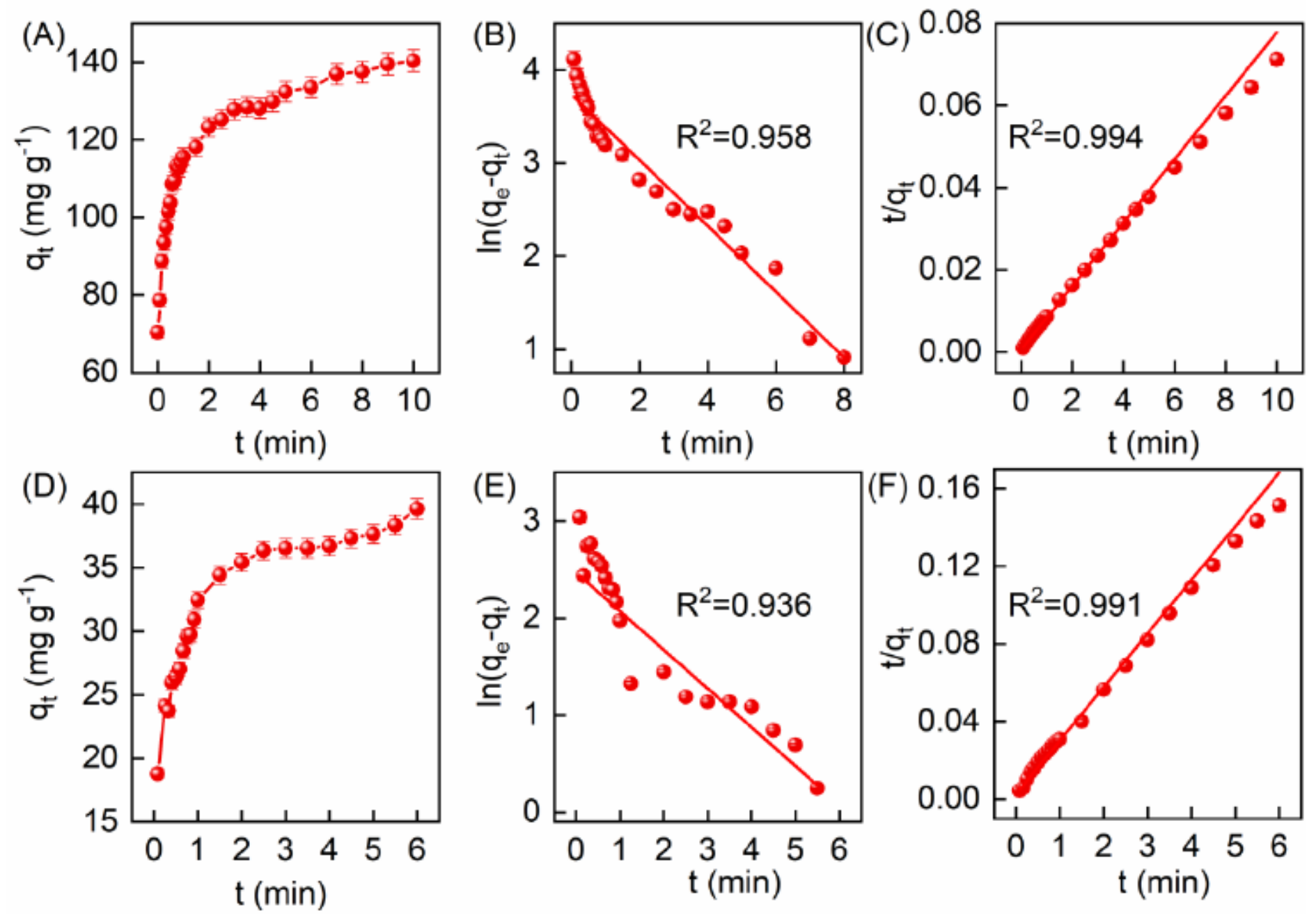

Figure 6

(A) The time-dependent adsorption of TCS by Co-NPC-800; (B) Quasi-first-order and (C) Quasi-secondorder kinetic curve for the adsorption of TCS by Co-NPC-800; (D) The time-dependent adsorption of PCMX by Co-NPC-800; (E) Quasi-first-order and (F) Quasi-second order kinetic curve for the adsorption of PCMX by Co-NPC-800. 
(A) The effect of temperature on TCS adsorbed by Co-NPC-800; Adsorption isotherms of TCS by Co-NPC800 at different temperatures; (B) Langmuir model isotherms fitted by Co-NPC-800 adsorbed TCS at different temperatures; (C) Freundlich model isotherms fitted by Co-NPC-800 adsorbed TCS at different temperatures; (D) The effect of temperature on PCMX adsorbed by Co-NPC-800; (E) Langmuir model isotherms fitted by Co-NPC-800 adsorbed PCMX at different temperatures; (F) Freundlich model isotherms fitted by Co-NPC-800 adsorbed PCMX at different temperatures.

\section{Figure 8}

The infrared spectrum of Co-NPC-800 after adsorption of (A) TCS or (B) PCMX; The number of hydrogen bond donors and acceptors of (C) TCS or (D) PCMX at different pH.

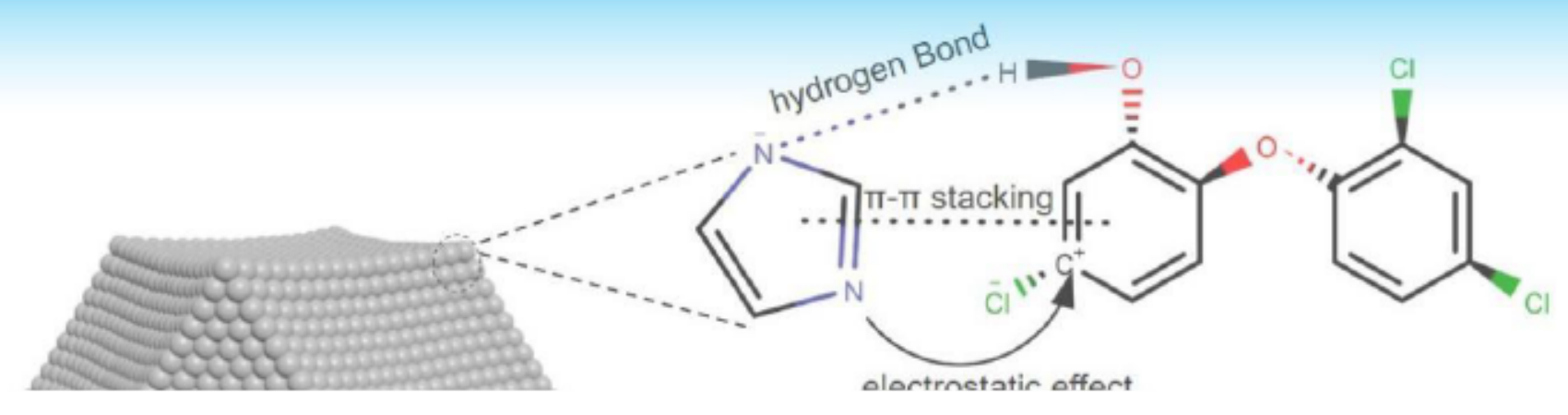

\section{Figure 9}

The adsorption mechanism of TCS and PCMX on Co-NPC-800. 


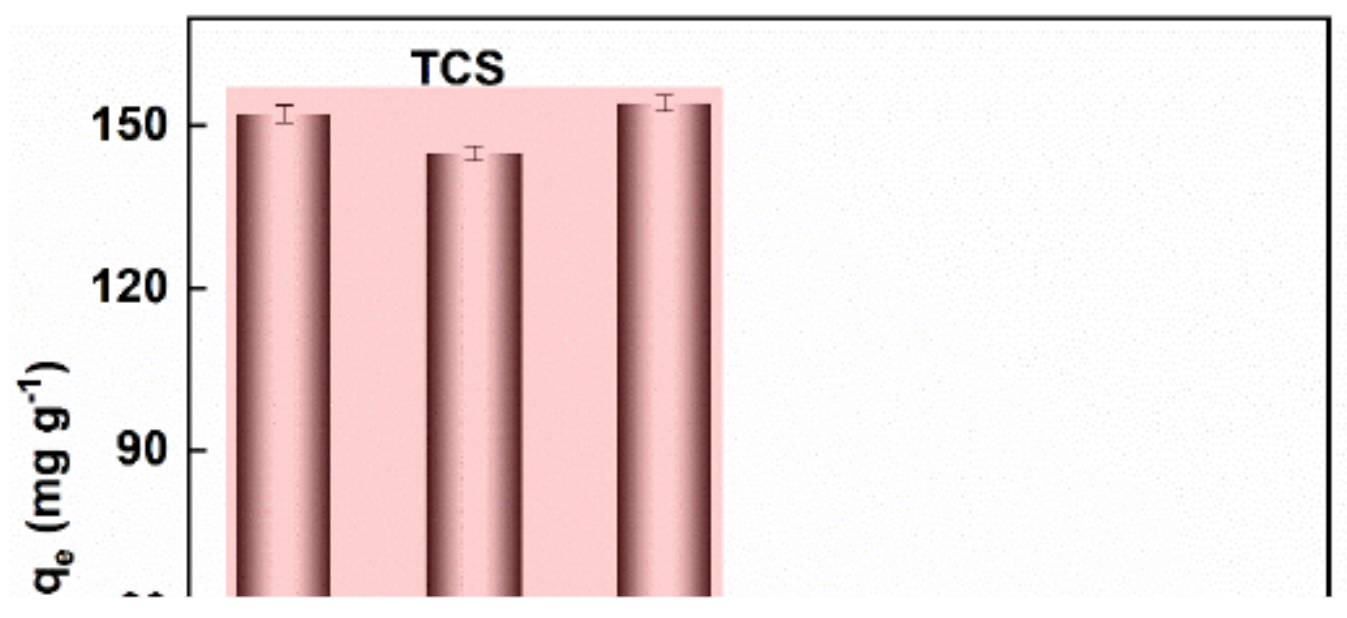

Figure 10

Co-NPC-800 application in the treatment of actual water samples

\section{Supplementary Files}

This is a list of supplementary files associated with this preprint. Click to download.

- SupportingInformation1021.docx 